\title{
Sur un cas de tétramérose hépatique du singe
}

\author{
par G. VASSILIADES, J. EUZEBY et N. GROSJEAN \\ [Laboratoire de Zoologie (Vers) associé au C.N.R.S., Muséum national d'Histoire naturelle \\ (Pr A.-G. Сhaвaud), 57, rue Cuvier, Paris, 5', \\ et Ecole nationale vétérinaire de Lyon, Laboratoire des maladies parasitaires]
}

\section{Résumé}

Un cas de tétramérose hépatique est décrit chez une guenon Mandrillus sp., dans le Puy-de-Dôme (France). L'espèce responsable Tetrameres fissispina (Diesing, 1861), Travassos, 1914, peut être considérée comme un «parasite transfuge ».

\section{Summary}

A case of hepatic tetrameriasis in a monkey Mandrillus sp. is reported and described in the Puy-de-Dôme (France). The species responsible for the disease, Tetrameres fissispina (Diesing, 1861) Travassos, 1914 may be considered as a «transfuge parasite ».

Le malade était une guenon mandrill (Mandrillus sp.) âgée d'environ trois ans et importée du Cameroun en juillet 1968.

Deux mois plus tard la guenon a été transportée par son acheteur dans la région du « Marais de Riom», dans le Puy-de-Dôme (France).

L'animal a présenté en novembre 1968 et de façon soudaine, un syndrome colique comportant l'émission de fecès sub-diarrhéiques mais surtout caractérisé par une très vive douleur dans l'aire hépatique et dans l'hypocondre droit ; cette crise violente s'est terminée par la mort, survenue après deux jours de maladie.

L'autopsie, pratiquée par le Docteur Strohl*, chef du laboratoire des Services vétérinaires du Puy-de-Dôme, a révélé l'existence d'une hépatite traumatique aiguë avec de très nombreuses travées hémorragiques parcourant le parenchyme du foie.

A partir d'un petit fragment de foie fixé dans une solution physiologique formolée à $8 \%$, l'examen microscopique de prélèvements opérés dans le tissu hépatique, a mon-

* Nous remercions très vivement le $D^{r}$ Strohl de nous avoir confié l'étude de ce cas et pour toute la peine qu'il a prise à en rassembler les commémoratifs. 
tré l'existence d'œufs sub-ellipsoïdes de $55 \mu$ sur $30 \mu$, à paroi épaisse et renfermant un embryon.

L'examen sur fond noir du produit de dilacération de la totalité du fragment hépatique a permis la découverte d'un exemplaire unique d'un nématode Spiruroidea mâle.

\section{Description de cet exemplaire.}

Corps long de $4,8 \mathrm{~mm}$ et large de $150 \mu$ au niveau du milieu du corps; pourvu d'épines cuticulaires réparties en quatre rangées sub-latérales: deux rangées latéroventrales de 72 à 75 épines, dont les dernières nettement ventrales, atteignent le niveau du cloaque ; deux rangées latéro-dorsales composées de 23 à 25 épines et se terminant à environ $1.400 \mu$ de l'extrémité antérieure. De chaque côté, entre la première épine latéro-dorsale et la première épine latéro-ventrale, sur la ligne latérale, on observe une épine supplémentaire (cf. fig. A et B).

Pharynx long de $25 \mu$ sur $8 \mu$ de large, œsophage musculaire et œsophage glandulaire longs respectivement de $325 \mu$ et $680 \mu$. l'apex.

Anneau nerveux et pore excréteur respectivement situés à $215 \mu$ et $280 \mu$ de

Deirides pourvues d'une épine fine, situées asymétriquement l'une à $150 \mu$, l'autre à $170 \mu$ de l'apex.

Queue longue de $150 \mu$ (distance de l'anus à l'extrémité postérieure du corps). Ailes caudales et papilles caudales absentes.

Sur la queue, on distingue ventralement deux rangées de cinq épines et latéralement, de part et d'autre de celles-ci, trois épines.

Deux spicules inégaux, spicule droit long de $125 \mu$, légèrement arqué à son extrémité, spicule gauche long de $380 \mu$, à pointe mousse, avec une sinuosité bien marquée à $70 \mu$ de la base (cf. fig. A).

Absence de gubernaculum.

Par les dimensions générales du corps et du tube digestif, par le nombre et la répartition des épines somatiques et caudales, par la forme et les dimensions des spicules, par les dimensions des œufs, cette espèce peut être identifiée à Tetrameres fissispina (Diesing, 1861) Travassos, 1914.

Cette espèce, parasite normal du ventricule succenturié des Ansériformes et des Galliformes, a pour hôte intermédiaire habituel un Crustacé Entomostracé dulçaquicole Daphnia pulex (ordre des Cladocères) ou Gammarus pulex (ordre des Amphipodes), c'est par l'ingestion de ces crustacés que la guenon a dû s'infester, probablement par souillure des aliments avec de l'eau contenant ces hôtes intermédiaires.

C'est sans doute après son arrivée dans le « Marais de Riom », région caractérisée par un système de drainage constitué d'un réseau de fossés dans lesquels l'eau circule à courant très faible et stagne souvent, et où canards et poules vivent en semi-liberté permanente, que l'infestation a pu se faire. 


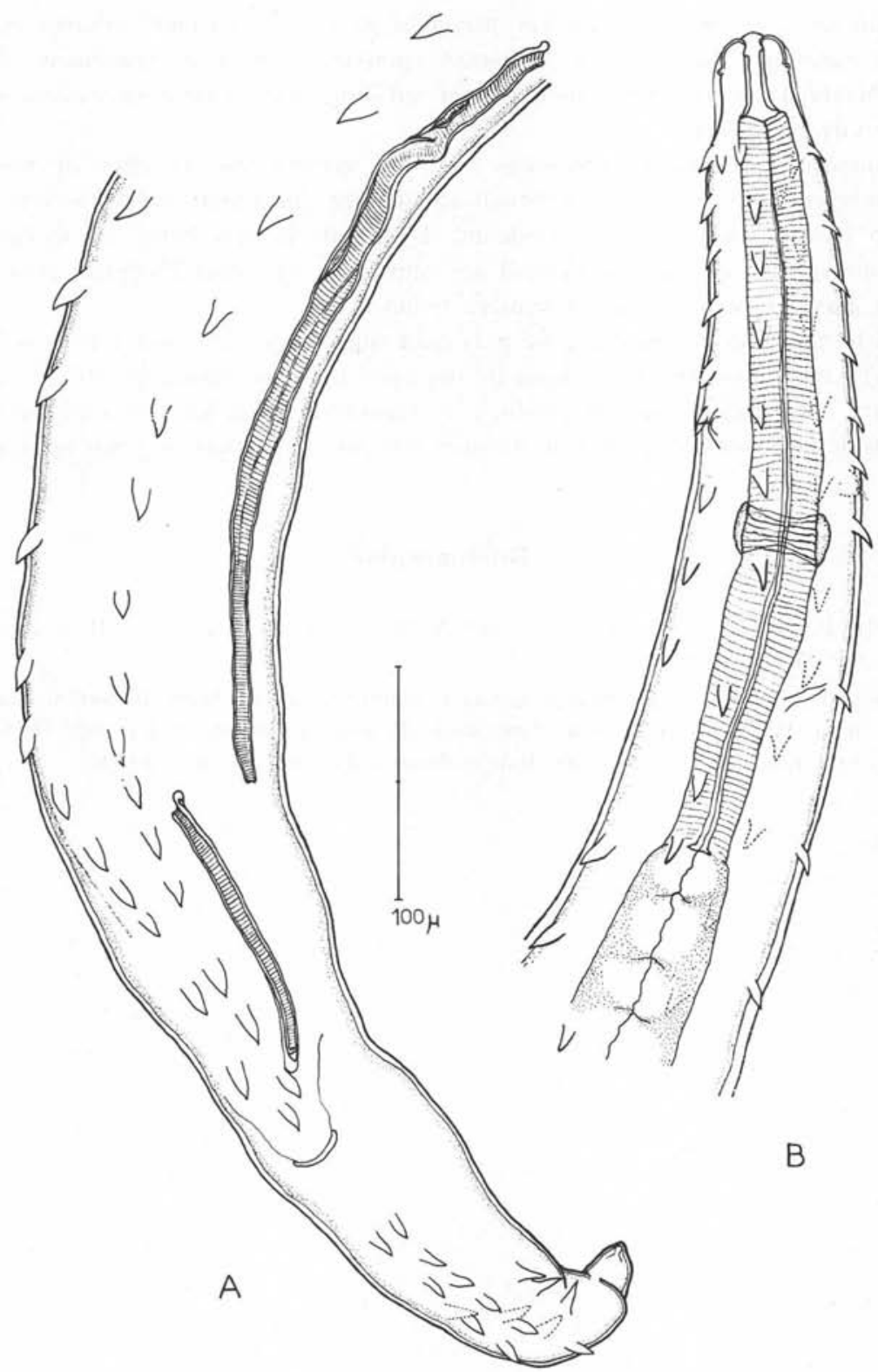

Il semble exclu en tout cas que la contamination ait pu s'opérer dans le pays d'origine de l'animal qui était en France depuis cinq mois, compte tenu de la rapidité de l'évolution de la maladie et de l'acuité des lésions hépatiques. 
Dans ce cas précis, Tetrameres fissispina peut être considéré comme étant un parasite transfuge, c'est-à-dire « un parasite pouvant évoluer complètement chez un hôte différent de l'hôte normal mais restant indistinguable morphologiquement » (Chabaud in Grassé, 1965).

L'intensité des lésions provoquées n'est pas surprenante ; en effet, de nombreux parasites tolérés par leurs hôtes habituels acquierrent une action pathogène très importante en passant chez un hôte accidentel. L'exemple le plus banal est le cas de la grande douve du foie, parasite normal des ruminants, qui, chez l'homme, peut provoquer de graves lésions, même en nombre réduit.

La tétramérose du singe décrite plus haut suggère que l'homme n'est pas à l'abri d'une telle contamination. L'ingestion de daphnies infestées naturellement est certainement rare mais doit pouvoir se produire accidentellement et les cliniciens n'étant pas prévenus de la possibilité d'une telle maladie, ont peu de chances de poser le diagnostic.

\section{Bibliographie}

Grassé (P.-P.), 1965. - Traité de Zoologie. Némathelminthes, t. IV, fasc. II, 731 pp. Masson et $\mathrm{C}^{\text {1e }}$ Editeurs, Paris.

Travassos (L.), 1914. - Contribuiçeos para o conhecimento da fauna helmintolojica brazileira. III. Sobre as especies brazileiras do genero Tetrameres Creplin, 1846. Mem. Inst. Oswaldo Cruz, Rio de Janeiro-Manguinhos, vol. 6, pp. 150-162. 